\title{
Normalisation of STEP Files for Improving the Data Compatibility of Transferred Tool Models
}

\author{
Claudia KLEINSCHRODT, Frank RIEG
}

\begin{abstract}
Due to the increasing digitalisation, the availability of high-quality data is gaining importance. However, the correct transfer of high-quality data across the entire supply chain is still a major problem. Studies show that by crossing system borders with neutral data formats, information is often distorted or even lost. An existing method, starting with setting up the requirements and carrying out compatibility tests, is refined and extended by an error weighting and used to analyse the problem and then determine the causes. On this basis, solution strategies can then be developed. As a promising solution to the normalization of STEP files turns out. This procedure is presented in more detail in this paper and examined by means of examples. Finally, the potential of this approach is evaluated and summarised.
\end{abstract}

Keywords: 3D model; data compatibility; STEP; tool industry

\section{INTRODUCTION}

In 2011 the initiative "Industrie 4.0" was introduced in Germany for the first time. This fourth industrial revolution is intended to expand the already highly automated industry by developing intelligent monitoring and autonomous decision-making processes to enable the control and optimisation of entire value-added networks [1, 2]. Keywords such as "internet of things", "cyberspace" or "digital factory" have shaped discussions in the media and politics. In order to realise these visions, a continuity of the information generated throughout the life cycle of a product is necessary. A prerequisite for this is the error-free transmission of the relevant data. Considering the manufacturing industry, the exchange of virtual tool models plays a decisive role. For example, for the management of server-based catalogues or the virtual commissioning of machines it is important to transfer the 3D models of the developers completely to the users [3].

A fundamental problem of many companies is the exchange of 3D CAD data. The transmission of information between different programs often corrupts or even loses the necessary data [4].

In order to assess the quality of the export or import of different 3D CAD programs, studies are conducted at the Chair of Engineering Design and CAD at the University of Bayreuth. In these investigations the transfer of different selected features via the neutral exchange format STEP is considered. These investigations are absolutely necessary because, in spite of the possibilities and the great potential of the STEP standard, studies show that there are often transfer problems in practice $[3,4]$. These errors result in multiple manual reworking of the virtual model and are preventing a development towards Industry 4.0.

The possible solutions are strongly dependent on the cause of the problem. This paper examines the avoidance of compatibility problems due to interface-related causes by normalising the STEP files.

\section{DATA TRANSFER OF 3D TOOL MODELS}

Data exchange is the foundation of a networked world. Through the global operating range of companies, complex supplier structures and media or system breaks within processes, the topic also gains relevance. This applies to all sectors in which data is generated. In these investigations the example of the tooling industry is used. Due to the complexity caused by the large number of tools, countless involved companies and various software used, the exchange of tool data has enormous potential for optimisation within the manufacturing industry.

\subsection{D Models in Tool Industry}

Virtual commissioning of single machine tools, machining simulations for process design or the virtual planning of complete production systems requires the virtual images of the real components, the so-called digital twins. These digital twins accompany their real model through their life cycle and contain all the information that the real component includes $[5,6]$

Depending on the future use, various demands are placed on the 3D CAD model of a tool. Committees of experts try to determine the necessary requirements to define them in standards. In Germany, the series DIN 4000 [7] and DIN 4003 [8] have been developed. The international description of the features is given in ISO 13399 [9]. In these standards the criteria such as the structure of the models, different anchor points or colour assignments are defined. These regulations are intended to standardise crucial references for further use while protecting the company-internal know-how and enable the developer to work creatively. Fig. 1 shows simplified example of a standard tool model for data transfer according to DIN 4003-82 [10].

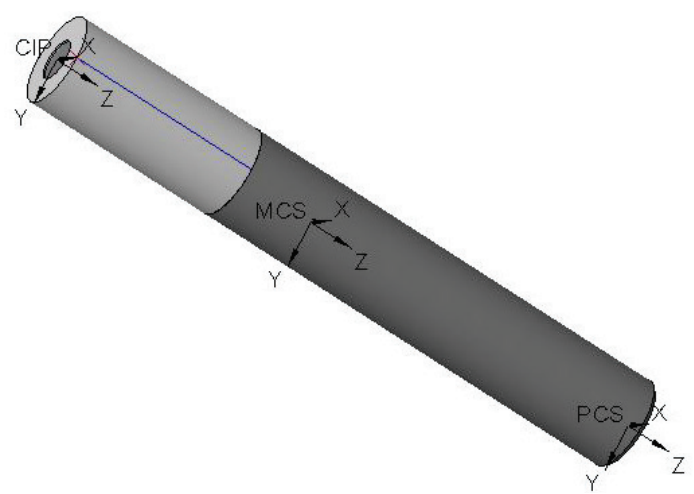

Figure 1 End mills for indexable inserts according to DIN 4003-82 
The 3D model, as its real model, will be sent to the customer, installed into virtual machine tools and integrated into digital processes. Therefore, the 3D CAD model has to be transmitted to different departments [6].

\subsection{Data Transfer of a 3D Model}

In general there exist two ways to transfer data between two systems: By using native, system-specific data or through conversion into neutral, systemindependent formats (Fig. 2) [11].

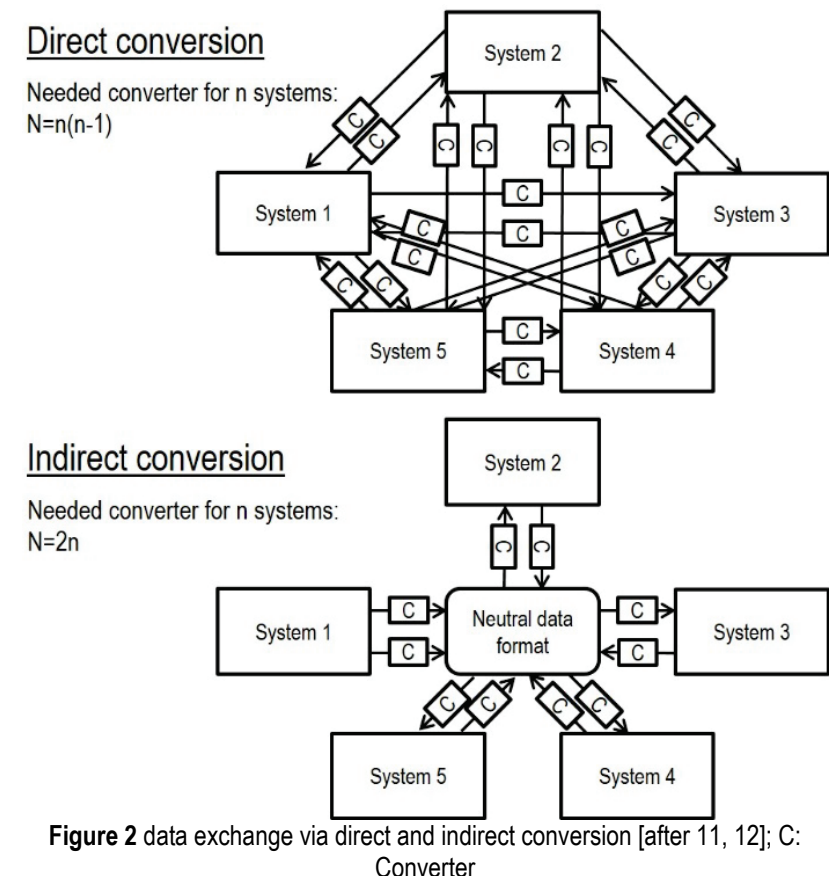

Due to the enormous system diversity of CAx software used in the industry, the virtual tool will have to bridge several system boundaries. Every system break causes a conversion of data and means a critical point in data continuity and completeness of information [11]. In direct conversion, interfaces are used which allow a direct translation of the start system's native format into the native format of the target system. In this case a maximum of the information can be transmitted. For each pairing, two direct converters are required, which lead to a converter number of $n \cdot(n-1)$ in the case of n systems. In view of the large number of systems used in the industry and the complexity of existing supplier structures, this approach is not intended. In practice, mostly neutral formats are used to exchange data. This method requires exactly two interfaces per system: one for importing and one for exporting the system-independent format. However, this type of data transfer often results in a corruption or even loss of data $[11,12]$.

\subsection{The Neutral Data Format STEP}

The STEP format (Standard for the Exchange of Product Model Data) takes a special value in the range of neutral formats for the transfer of 3D data. This format has existed as an ISO standard (ISO 10303) since 1992. From the outset it has been built as an extensive standard and nowadays it is implemented in almost every CAx program
[13]. The core of the STEP format is called Application Protocols (AP), which are responsible for the representation of various information in an exchange file $[11,13,14,15]$. AP 203 (Configuration controlled 3D designs of mechanical parts and assemblies) [16], AP 214 (Core data for automotive mechanical design processes) [17] and AP 242 (Managed Model-based 3D Engineering) [18] are the relevant application protocols to the tooling industry $[11,19]$. AP 214 is currently mainly used for data exchange because AP 203 is considered as obsolete and AP 242 has been published in 2014, so it is too recent to be comprehensively implemented [19]. This is why the following investigations will consider the data exchange via the neutral format STEP AP 214.

\subsection{State of the Art}

At the international level, organisations like the ProSTEPiViP association and the CAx Implementer Forum are focused on the general problem of data exchange of 3D models via different standards. Largescale benchmark tests are used to identify the deficiencies of different interfaces and to develop solutions. In these studies, test objects from various systems were exported and then imported again, in order to assess the quality of the STEP processors. The criteria used in these test rounds change frequently and are for example a correct transfer of volume, surface content or certain display properties. The resulting documents, such as guidelines and recommendations, are then available to the members. ProSTEPiViP discontinued the studies on STEP AP 214 in 2003. The CAx Implementer Forum continues the investigations for AP 214 [20, 21].

Due to the use of changing systems, different versions and varying requirements at different test rounds over a long period of time, the individual results are not systematically comparable with each other and thus cannot be checked against current studies. In order to establish a comprehensive comparability, it is important to use a uniform test method. Furthermore, changing factors, such as exact system versions, must be documented in a way that is accessible to all interested parties.

\section{METHOD FOR ANALYSING AND IMPROVING THE DATA QUALITY}

In [22] a four-step method to consider the problem of insufficient quality of the data transfer is described:

1) Definition of data exchange requirements

2) Compatibility checks and exhaustive analysis of the exchange results

3) Classification of the problems by their cause

4) Development of solution strategies depending on the classification.

This procedure is extended by an error weighting and elaborated in detail as shown in Fig. 3.

\subsection{Requirements for Tool Data Exchange}

Depending on the sector and further use of the models, different requirements are placed on the exchange result. Just for the pure representation of the $3 \mathrm{D}$ model, for 
example for marketing purposes, the transfer of the external appearance is sufficient. For additional functional applications such as simulations or automated data storage, higher demands are placed on the models. The German tooling industry has agreed on common transfer characteristics in the series of standards DIN 4000 and DIN 4003. These standards define various anchor points such as the primary coordinate system (PCS) or the cutting reference point (CRP). In addition, colours with defined
RGB (red green blue) values are used for the function assignment of individual components to allow an automated interpretation. For instance, the non-cutting part is coloured with a dark grey (RGB: 127/127/127), the cutting part with a light grey (RGB: 204/204/204) and the cutline with blue (RGB: 0/0/255). Depending on the classification of a tool other attributes are claimed in the standard $[7,8]$.

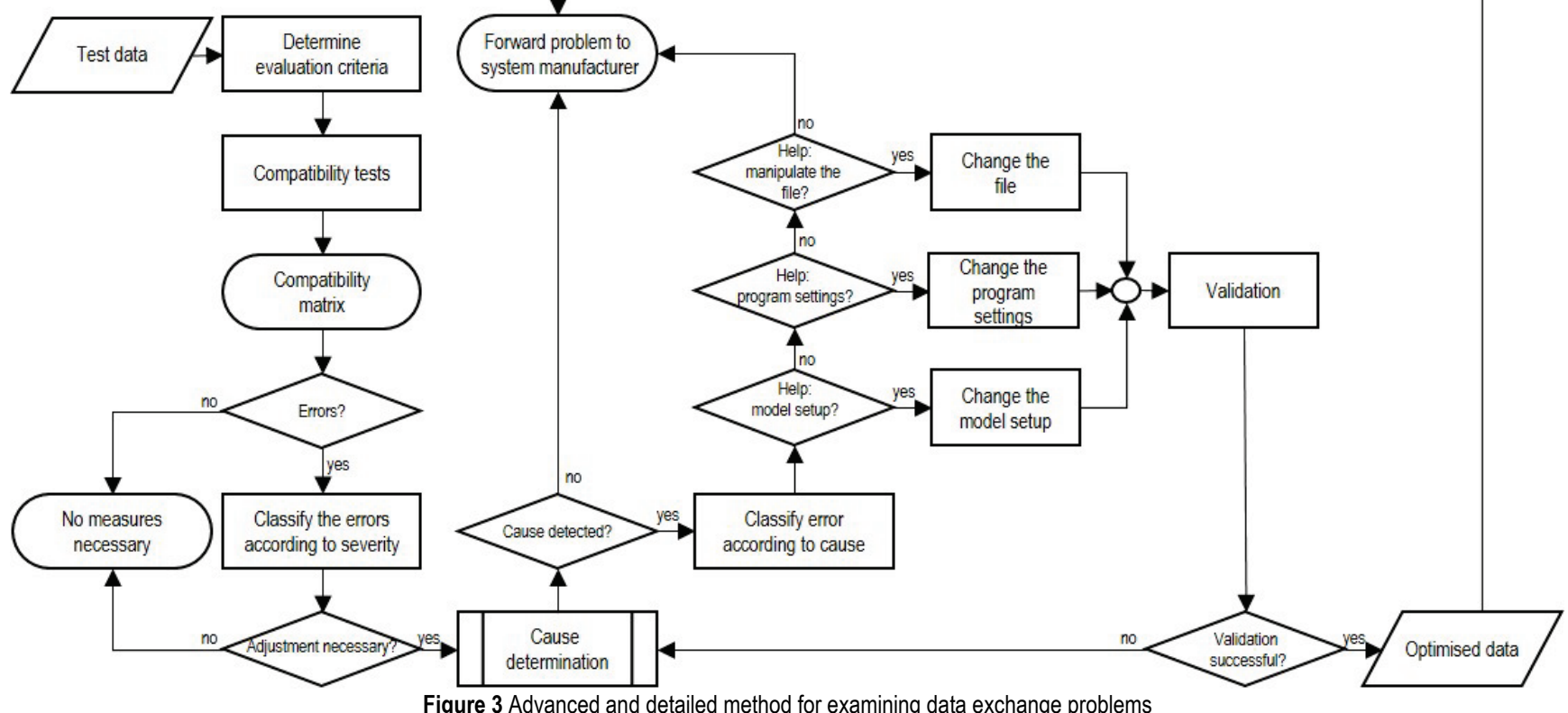

In order to generate universal results for the tooling industry as well as across all sectors, the characteristics required in the series of standards are abstracted. Coordinate systems, levels, points, axes, sketches and annotations serve as verification features. The colour values defined in the standards are used as controlled colours. The characteristics to check are selected depending on the demand and the models to be tested.

\subsection{Compatibility Checks - an Example}

In order to improve the quality of exchanged data, the second step is to identify the points of failure. An effective method for this is the implementation of compatibility checks in which files (exported from numerous systems) are reimported by several systems. This approach is based on existing methods of the ProSTEPiViP association and the CAx Implementer Forum. Nevertheless, a comparison with these studies is avoided because of the reasons mentioned in chapter 2.3 .

Tab. 1 shows a resulting test matrix. In this case the components were created by four different companies in their standard system and exported as STEP-file. The CAD software systems used are CREO 3.0 (PTC), CATIA V5 (Dassault Systèmes) and NX 10 (Siemens). In this analysis, the colour values, which are defined in DIN 4003, were verified. When looking at the matrix, it is noticeable that the exported data of two companies (B and D) do not contain information about the colour blue. So it is logical that this colour does not appear in the imported models. However, company D uses the same software as company $\mathrm{C}$ and its file contains the necessary information. It can also be determined that software 3 switches the RGB value of the dark grey from $127 / 127 / 127$ to $125 / 125 / 125$. The deviations of the colour values are still within the tolerance, nevertheless, a falsification took place.

Table 1 Compatibility check of STEP-files, which were exported from different companies using different software systems (considering colour transfer)

\begin{tabular}{|c|c|c|c|c|c|c|c|c|c|c|c|c|}
\hline & \multirow{2}{*}{\multicolumn{3}{|c|}{ Export }} & \multicolumn{9}{|c|}{ Import } \\
\hline & & & & \multicolumn{3}{|c|}{ Software 1} & \multicolumn{3}{|c|}{ Software 2} & \multicolumn{3}{|c|}{ Software 3} \\
\hline Software 1 (Company A) & + & + & + & + & + & + & + & + & - & + & 0 & - \\
\hline Software 2 (Company B) & + & + & - & + & + & - & + & + & - & + & $\bullet$ & - \\
\hline Software 3 (Company C) & + & + & + & + & + & + & + & + & + & + & $\bullet$ & + \\
\hline Software 3 (Company D) & + & + & - & + & + & - & + & + & - & + & $\bullet$ & - \\
\hline
\end{tabular}

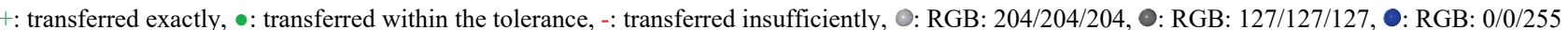

To validate the results of the compatibility tests by using original firm data the tests are repeated with a self- generated representative test object. The results of this study are shown in Tab. 2. Several problems can be solved 
by using adjusted settings and a suitable model construction. However, the insufficient colour transfer of the colour blue from software 1 to the other two systems as well as an incorrect representation of the dark grey in software 3 remain.

Table 2 Compatibility check of STEP-files of a representative test object (considering colour transfer)

\begin{tabular}{|c|c|c|c|c|c|c|c|c|c|c|c|c|}
\hline & \multirow{2}{*}{\multicolumn{3}{|c|}{ Export }} & \multicolumn{9}{|c|}{ Import } \\
\hline & & & & \multicolumn{3}{|c|}{ Software 1} & \multicolumn{3}{|c|}{ Software 2} & \multicolumn{3}{|c|}{ Software 3} \\
\hline Software 1 & + & + & + & + & + & + & + & + & - & + & $\bullet$ & - \\
\hline Software 2 & + & + & + & + & + & - & + & + & + & + & - & + \\
\hline Software 3 & + & + & + & + & + & + & + & + & + & + & • & + \\
\hline
\end{tabular}

: transferred exactly, $\bullet$ : transferred within the tolerance, -: transferred insufficiently, ○: RGB: 204/204/204, ○: RGB: 127/127/127, ๑: RGB: 0/0/255

Another study considers the transfer of different structural elements by using a representative test object (Fig. 4). This 3D model in total contains 34 constructive attributes, like coordinate systems or planes, and eight colours for checking the quality of data transfer. The features are based on the requirements listed in DIN $4000 / 4003$.
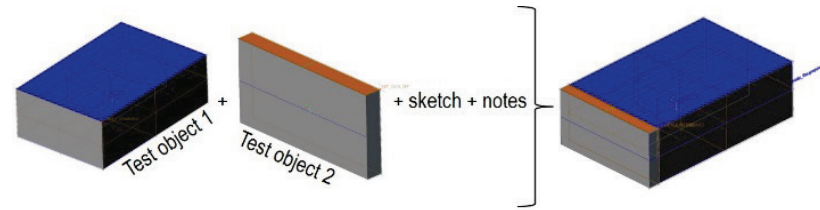

Figure 4 Representative test object with 34 constructive attributes and eight colours, based on the requirements listed in DIN 4000/4003

In this study CAD software systems used are CREO 3.0 (PTC), CATIA V5 (DassaultSystèmes), NX 10 (Siemens) and SOLIDWORKS 2015 (DassaultSystèmes). The test object is designed separately in all considered CAD systems. The import and export is performed with the previously determined optimal processor settings. Subsequently the result is regarded in the four CAD systems used. The evaluation of the data transfer of the constructive attributes is shown in Tab. 3. The overall overview shows that on average just $40 \%$ of the tested components were transferred. The quality between the programs deviates strongly. Software 1 can handle $46 \%$ of the features, software 2 even $62 \%$. In software 3 only $28 \%$ and in software 4 only $24 \%$ of the features were imported. In particular, also the problems of individual software systems with transmitted information can be seen in this table. For instance, software 3 is not capable of displaying any planes and software 4 ex- and imports only one coordinate system per component. Even though the described studies are just examples, it is clear that there are a lot of problems which prevent an automatic data transfer. By considering other software systems and features described in the standards, the problems can be characterised more precisely and solutions can be identified.

Table 3 Compatibility check of STEP-files of a representative test object (considering construction characteristics)

\begin{tabular}{|c|c|c|c|c|c|c|c|c|c|c|c|c|c|c|c|c|c|c|c|c|c|c|c|c|c|c|c|c|c|c|c|c|c|}
\hline \multicolumn{20}{|c|}{ Test object 1} & \multicolumn{9}{|c|}{ Test object 2} & \multicolumn{5}{|c|}{ Assembly } \\
\hline \multicolumn{3}{|c|}{$\mathrm{CS}$} & \multicolumn{8}{|c|}{$\mathrm{PL}$} & \multicolumn{2}{|c|}{$\mathrm{P}$} & \multicolumn{6}{|c|}{$\mathrm{A}$} & $\mathrm{S}$ & $\mathrm{CS}$ & \multicolumn{6}{|c|}{ PL } & \multicolumn{2}{|c|}{$\mathrm{S}$} & \multicolumn{3}{|c|}{ PL } & $\mathrm{S}$ & $\mathrm{N}$ \\
\hline 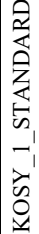 & $\begin{array}{l}-\overrightarrow{1} \\
\vec{n} \\
0 \\
\underline{v}\end{array}$ & $\begin{array}{l}N_{1} \\
\lambda^{\prime} \\
0 \\
0\end{array}$ & 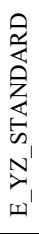 & 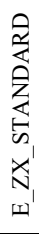 & 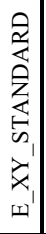 & $\mid$\begin{tabular}{|l}
$\overrightarrow{1}$ \\
\multicolumn{1}{|c}{$\mid$}
\end{tabular} & $\begin{array}{l}N_{1} \\
\text { 山r }\end{array}$ & $\begin{array}{l}N \\
N_{1}\end{array}$ & $\left|\begin{array}{l}x \\
x_{1} \\
-1\end{array}\right|$ & $\begin{array}{l}\vec{x} \\
x_{1}\end{array}$ & $\left|\overrightarrow{a_{1}}\right|$ & $\begin{array}{l}N_{1} \\
n_{1}\end{array}$ & $\begin{array}{l}x_{1} \\
\ll_{1}\end{array}$ & $\mid \begin{array}{l}\lambda_{1} \\
<\end{array}$ & $\begin{array}{l}N_{1} \\
\ll_{1}\end{array}$ & 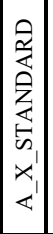 & 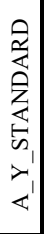 & 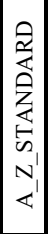 & 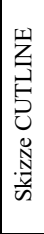 & 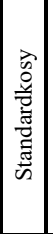 & $\left|\begin{array}{l}\frac{7}{60} \\
\vec{a} \\
\overrightarrow{2}\end{array}\right|$ & ने & 泀 & $\left|\begin{array}{l}x_{1} \\
x_{1} \\
y_{1}\end{array}\right|$ & $\left|\begin{array}{l}N \\
X \\
\\
=\end{array}\right|$ & $\mid \begin{array}{l}\varkappa_{厶} \\
\mathbf{N}_{1}\end{array}$ & 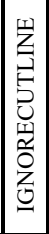 & 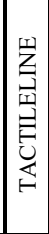 & 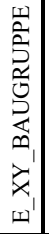 & 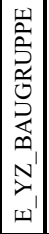 & 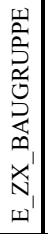 & 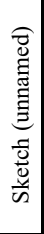 & 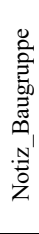 \\
\hline
\end{tabular}

Compatibility to Software 1

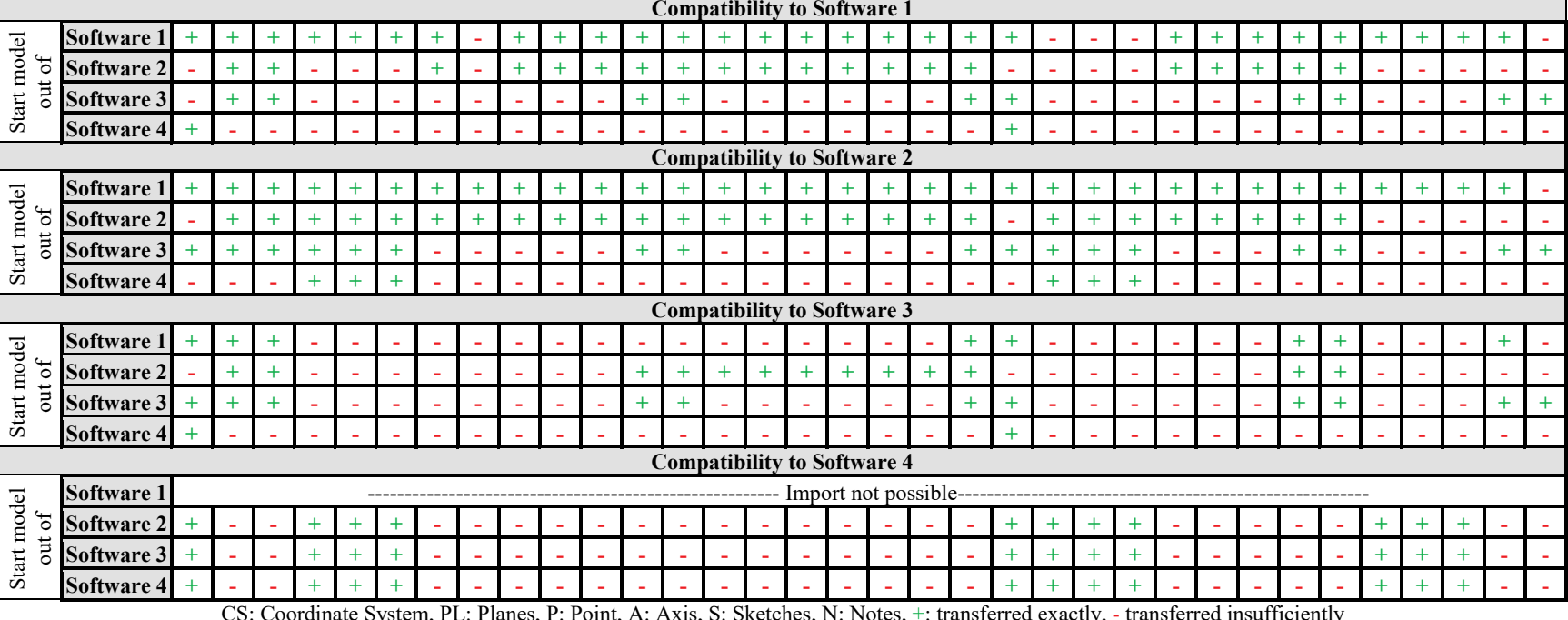




\subsection{Error Weighting}

Occurring quality defects that are revealed by the compatibility tests can be evaluated by severity depending on the later application. For this purpose, refined evaluation criteria are set up, which are specifically tailored to the following use. These criteria can be modified additionally with weighting factors (WF) and then applied to the various errors.

In the example of color transmission from Tab. 2 and Tab. 3, the presence of the CUT and NOCUT area as well as the CUTLINE (WF: 3), the colouring of areas and sketches according to the tolerance limits described in DIN 4003-1 (WF:2) and the exact RGB colouring of the areas and sketches (WF: 1) can be used as evaluation criteria.

If these criteria are now applied to the errors that occur in this example, then the missing CUTLINE is rated as a serious error, the decolourisation of the CUTLINE as medium error and the color shift of the NOCUT area in software 3 as a slight error. This rating can aid in prioritising the needs of action.

\subsection{Classification of the Problem Sources}

The comparative tests carried out allow a characterisation of the problems. When considering all conducted studies, the problems can be classified depending on their causes as follows:

- User-related problems

- Ambivalence of the standard interface STEP

- System specific inconsistency

The influence of the user on the exchange result is shown for example in Tab. 1: Although company $\mathrm{C}$ and $\mathrm{D}$ use the same software, they achieve other transfer results. The causes for this do not lie in the software or the interface, but in individual decisions of the design engineers. This can be the construction of the 3D model or the used export settings. Depending on the program and configuration, various settings can be made during the export or import, which have a crucial influence on the transfer result. However, the user usually does not have the experience, the knowledge or the needed access rights to choose these settings properly.

The use of standard interfaces should allow the transfer of information over system boundaries. The format STEP for example is defined in international norms $[11,13]$. Despite this uniform definition, the standard leaves enough scope for an ambiguous description of 3D models. Thus it is possible that STEP files, which are conform to the standard, are not compatible due to different interface implementations.

This can be clarified using Tab. 2. Although optimal settings and a very simple test object have been used, correct transfer of the colour blue outgoing from system 1 is not possible. If some entities are added in the STEP file (as shown in below), a correct transferred display of the colour values is achieved. So this kind of problem is caused by the ambivalence of the standard interface STEP.

\#75=COMPOSITE_CURVE(",(\#47,\#56,\#65,\#74),.F.); \#182=CURVE_STYLE(",\#21,POSITIVE_LENGTH_ME ASURE(2.E-2),\#1);
\#183=PRESENTATION STYLE ASSIGNMENT((\#182 ) ); \#184=STYLED ITEM(",(\#183),\#75); \#185=MECHANICAL_DESIGN_GEOMETRIC_PRESE NTATION_REPRESENTATION(",(\#38,\#46,\#55,\#64,\#7 3,\#184),\#90);

Problems that occur exclusively in one used software can often be attributed to system-specific properties. An example is the transfer of the dark grey colour into system 3 as it is shown in Tab. 2. Although the colour values in the STEP file are correct, the colour is always displayed with slightly changed colour values. This occurs independently of the output system. The problem is caused by the standard colour settings of the target system. Instead of the transferred colour, the software uses the next standard colour of the system-specific colour palette.

\subsection{Extractive Solutions}

Just as the causes of transfer problems are manifold, solutions also have to be implemented in different ways. These can be summarised as three opportunities of intervention for the user:

- Change procedure during model construction

- Adapt program settings

- Manipulate the exported files

User-specific problems can only be solved by supporting and training system users. Firstly, all users of CAx software must be sensitised to the problem of data exchange and the challenges of a digitised world. When this is achieved, recommendations for the respective settings for import and export depending on the used system combinations help to provide an overview. Company-internal configurations with fixed system settings can avoid problems in standardised processes. Furthermore, designers have to be trained on a standardcompliant design of their models. In order to be able to identify problems more easily by the system user, check routines are created, which check the STEP files for standard conformity and compatibility.

By skilful handling of the user, sometimes interfacerelated problems can be avoided. The remaining incompatibilities caused by the interfaces can be prevented, for example, by specifically manipulating the STEP physical file (as shown in Fig. 4).

The system-specific problems can only be solved by the software developers themselves. They only have the possibility to implement changes in their software. Industry and research have the responsibility to get the developers' attention for mistakes and possible solutions.

\section{NORMALISATION FOR IMPROVING THE DATA COMPATIBILITY}

By directly manipulating the STEP files, compatibility problems arising during the exchange can be eliminated even without the intervention of users or system developers. One way to increase the general compatibility of STEP files is the normalisation of the files. 


\subsection{Procedure for the Normalisation of STEP Files}

By normalising STEP files, different system-specific representations of the same components can be compensated. The first is to find out how the individual CAD software constitute several modules.

For this purpose, minimal models of the elements are created for each program. These files are created by generating the component in the program and exporting it as STEP. Afterwards, all elements which are not necessary for the correct display in the program are removed. So these contain only the entries which are absolutely necessary for the representation of the component in the respective software. Since the necessary entries for the description of the elements in their base are usually the same and only individual entries are displayed, the entities can supplement each other. In this way, a master model is created that is compatible with all included programs.

\subsection{Example for Improving the Data Compatibility by Normalisation: Point}

The procedure described in chapter 4.1 is now applied to a simple example. As test element a point is used, which is described in the STEP file as followed:

$\# 1=$ CARTESIAN POINT('P 0',(0.,0.,0.));
Three different CAD systems (CREO 3.0 (PTC), CATIA V5 (Dassault Systèmes), NX 10 (Siemens)) are considered for which minimal models of the element "point" are created. The point itself is displayed the same way each time, but the different programs show different additional entries in the data section, as Tab. 4 shows. Based on these minimum models, a master model can be created that combines the required entries of all systems (Tab. 4).

In order to examine the exchange quality of the generated system-influenced minimal files as well as of the normalised master model, a compatibility test as described in chapter 3.2 is carried out (Tab. 5). The three generated minimal models as well as the master model were imported into the three considered CAD systems. Software 1 reacts most tolerant to the imported files due to the low number of necessary entries. Software 2 requires the entity "GEOMETRICALLY BOUNDED SURFACE SHAPE REPRESENTATION" to display and name the component. In contrast, software 3 needs just some kind of "SHAPE REPRESENTATION" and thus can also represent the minimal model of software 2 correctly.

While the minimum models generated from software 1 and 3 show deficits in data transmission, the minimal model out of software 2 and the master model can be imported without any errors. So in this particular case, it would also be possible to use the minimal model out of system 2 as master model. However, this would hamper the generality of the approach, as the next example shows.

Table 4 Minimal models in three different CAD systems are merged into a STEP-master model

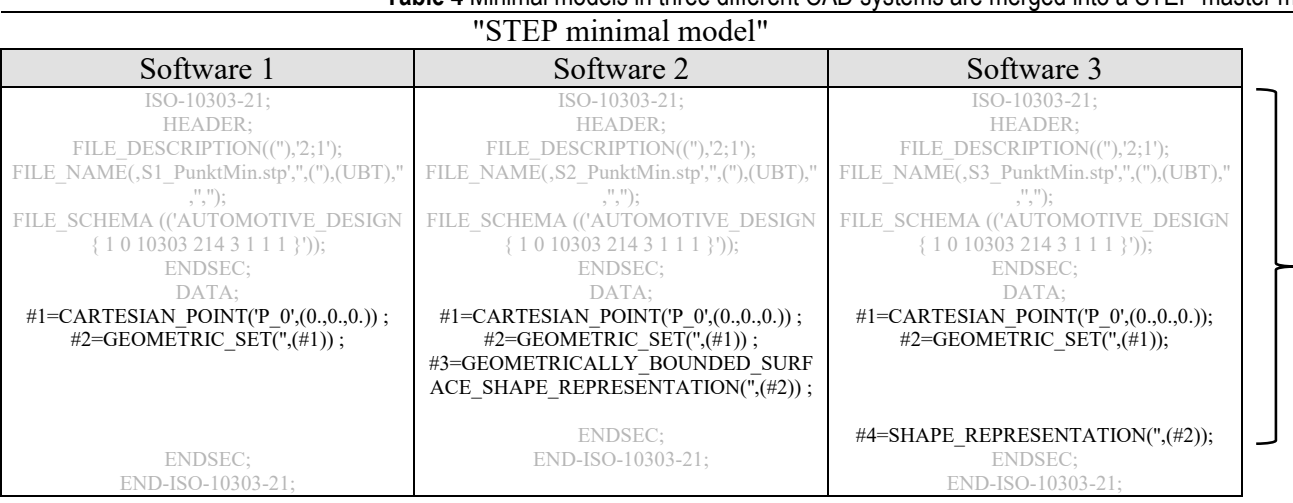

"STEP mastermodel"

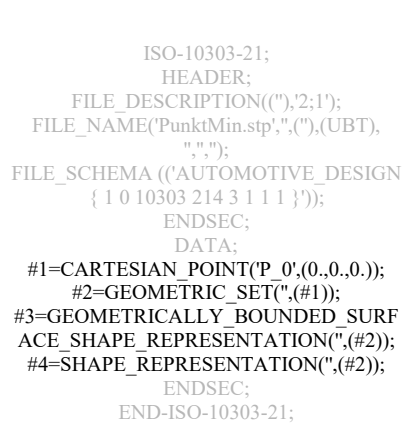

Table 5 Compatibility test of the minimal models and the generated master model

\begin{tabular}{|c|c|c|c|c|c|c|c|c|c|c|}
\hline & & \multicolumn{9}{|c|}{ Import in } \\
\hline & & \multicolumn{3}{|c|}{ Software 1} & \multicolumn{3}{|c|}{ Software 2} & \multicolumn{3}{|c|}{ Software 3} \\
\hline & & I & $\mathrm{D}$ & $\mathrm{N}$ & I & $\mathrm{D}$ & $\mathrm{N}$ & I & $\mathrm{D}$ & $\mathrm{N}$ \\
\hline \multirow{3}{*}{ Minimal model out of } & Software 1 & + & + & + & + & - & - & - & - & - \\
\hline & Software 2 & + & + & + & + & + & + & + & + & + \\
\hline & Software 3 & + & + & + & + & - & - & + & + & + \\
\hline \multicolumn{2}{|c|}{ Master model } & + & + & + & + & + & + & + & + & + \\
\hline
\end{tabular}

I: Import, D: Display, N: Naming, +: transferred sufficiently, -: transferred insufficiently

\subsection{Example for Improving the Data Compatibility by Normalisation: Coordinate System}

In this example, the described procedure is repeated using a coordinate system. Again, the same three systems are used and minimal models as well as the master model are derived (Tab. 6). In comparison, the file out of software 3 has much more entries than the other. In addition, all entries of the other files are included. In this example the compatibility test (Tab. 7) shows that the files of programs
1 and 2 produce errors during the import, while the minimal model of software 3 as well as the master model can be transferred correctly. Thus, in this case the minimal model of software 3 could be used as the master model.

\subsection{Quality Improvement through Normalisation of the STEP Files}

In both examples shown, a considerable improvement in the exchange quality could be achieved. 
While in the compatibility tests with the minimal models only $74 \%$ (point) and $70 \%$ (coordinate system) of the tested features could be transferred, both Master STEP models show transmission rates of $100 \%$.

In each example, there is also a system that is capable of producing files of sufficiently high quality. However, these are different systems: in the first example, software 2 shows the best results, in the second example it is software 3. In order to be able to transfer models with different components in general, the master models created by normalisation must be used.

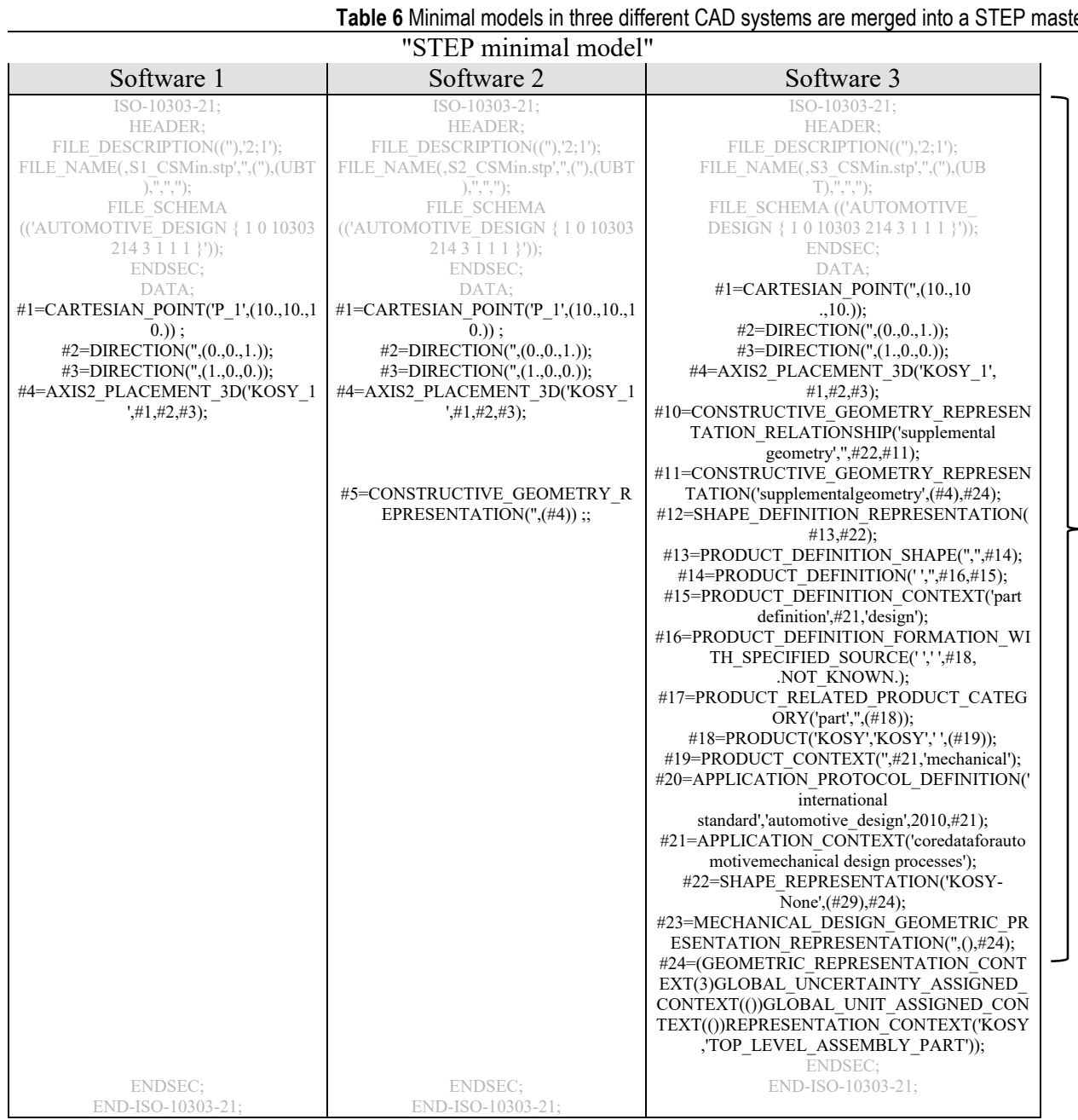

"STEP mastermodel"

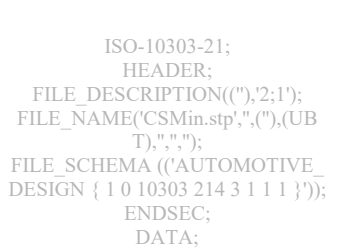

\#1=CARTESIAN_POINT(",(10.,10 $., 10$.$) );$

\#2=DIRECTION(",(0.,0.,1.));

\#2=DIRECTION(",",(1.,0.,0.)) \#4=AXIS2_PLACEMENT_3D('KOSY_1', $\# 1, \# 2, \# 3)$;

\#10=CONSTRUCTIVE GEOMETRY REPRESEN TATION RELATIONSHIP('supplemental TATION_RELATIONSHIP('supp

geometry',",\#22,\#11);
\#11=CONSTRUCTIVE GEOMETRY REPRESEN TATION('supplementalgeometry',(\#4),\#24); \#12=SHAPE_DEFINITION_REPRESENTATION $\# 13, \# 22)$;

\#13=PRODUCT_DEFINITION_SHAPE(",",\#14); \#14=PRODUCT DEFINITION(' ',",\#16,\#15); \#15=PRODUCT_DEFINITION_CONTEXT(

\#16=PRODUCT DEFINITION FORMATION WI

TH_SPECIFIED_SOURCE(' ',' ',\#18, \#17=PRODUCT_RELATED_PRODUCT_CATEG

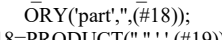

\#18=PRODUCT(",,", ',(\#19)); \#20=APPLICATION_PROTOCOL_DEFINITION(' international

standard','automotive design',2010, \#21);

\#21=APPLICATION CONTEXT(");

\#22=SHAPE_REPRESENTATION(',(\#29),\#24);

\#23=MECHANICAL DESIGN GEOMETRIC PR ESENTATION_REPRESENTATION(",(),\#24); \#24=(GEOMETRIC_REPRESENTATION_CON EXT(3)GLOBAL_UNCERTAINTY ASSIGNED CONTEXT $(())$ GLOBAL_UNIT ASSIGNED CON TEXT(O)REPRESENTATION CONTEXT( ',TOP LEVEL ASSEMBLY PART'));

Table 7 Compatibility test of the minimal models and the generated master model

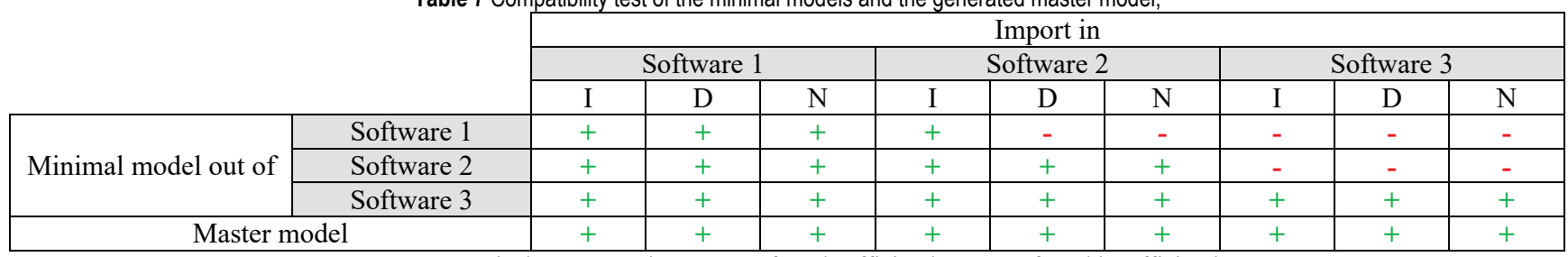

I: Import, D: Display, N: Naming, +: transferred sufficiently, -: transferred insufficiently

\section{CONCLUSION}

The poor quality of exchanged 3D models still poses a big problem in today's industry. Many processes are slowed down and a completely digitised and automated smart factory or Industry 4.0 is not yet feasible.

In order to tackle the problem of defective quality of exchanged tool data, a structured approach must be taken.

Therefore, the method described in [22] is extended by an error weighting and detailed for analysing several examples. The requirements are determined according to DIN 4000/4003 and compatibility tests are carried out, which reveal many problems in the data exchange. By using the added error weighting problems can be prioritised according to their need of action. The associated classification can group the problems into user-related, interface-related and system-related errors. The determined causes can be used directly to avoid errors. However, cross-linking is also possible. For example, many user-related problems are fixed by file manipulation. There are solutions by changing the model structure, adapting the program settings or manipulating the exported files. In this paper the normalisation of STEP files to increase compatibility is investigated and applied.

In the examples from chapters 4.2 and 4.3 , it was shown clearly that different CAD systems have different 
requirements regarding the structure of STEP files. The content and scope of the derived minimal models can differ strongly. In both examples, one file was found to be just as compatible as the master model. However, since they are files created by different systems, it is not appropriate for a general solution to use them for optimising the compatibility. The more general approach is the creation of master models, which proved successful in all tests carried out. This is therefore a promising approach, which should be pursued further to achieve an improvement in exchange data quality, since data exchange is the key for Industry 4.0.

\section{REFERENCES}

[1] Kagermann, H., Lukas, W. D., \& Wahlster, W. (2011). Industrie 4.0: Mit dem Internet der Dinge auf dem Weg zur 4. industriellen Revolution. VDI Nachrichten, 13, 11.

[2] Bundesministerium für Bildung und Forschung (Ed.). (2012). Bericht der Bundesregierung. Zukunftsprojekte der Hightech-Strategie (HTS-Aktionsplan). Bonn: W. Bertelsmann Verlag.

[3] Kleinschrodt, C., Hackenschmidt, R., \& Rieg, F. (2015). Evaluierung des 3D-Datenaustauschs von Präzisionswerkzeugen. In Brökel, K., Feldhusen, J., Grote, K.-H., Müller, N., Rieg F., \& Stelzer, R. (Eds.). 13. Gemeinsames Kolloquium Konstruktionstechnik, Clausthal, 79-88.

[4] Kleinschrodt, C. (2016). Industrie 4.0 in Gefahr - scheitert die vierte industrielle Revolution am Datenaustausch? Werkzeug Technik, 156, 143-145.

[5] Siemens AG (2015). Digitalisierung im Maschinenbau. Advance. Digital Enterprise - auf dem Weg zu Industrie 4.0. 2, 4-13

[6] Stark, R., Damerau, T., \& Lindow, K. (2016). Industrie 4.0 Digitale Neugestaltung der Produktentstehung und Produktion am Standort Berlin. In Industrie 4.0 grenzenlos, Berlin, Heidelberg: Springer Vieweg, 169-184.

[7] DIN 4000-1:2012-09: Tabular layouts of properties - Part 1: Concepts and principles. Berlin: Beuth.

[8] DIN 4003 -1:2014-02: Concept for the design of 3D models based on properties according to DIN 4000 - Part 1: Overview and fundamental principles. Berlin: Beuth.

[9] ISO 13399-1:2006-02: Cutting tool data representation and exchange - Part 1: Overview, fundamental principles and general information model. Geneve: ISO

[10] DIN 4003 -82:2013-08: Concept for the design of 3D models based on properties according to DIN 4000- Part 82: End mills with non-indexable cutting edges. Berlin: Beuth.

[11] Troll, A. (2011). CAx-Datenaustausch mit neutralen Datenformaten: prozessgetriebene Konzeption eines Assistenzsystems für die Produktentwicklung. Aachen: Shaker.

[12] Vajna, S., Weber, C., Bley, H., \& Zeman, K. (2009). CAx für Ingenieure: eine praxisbezogene Einführung. Berlin Heidelberg: Springer-Verlag.

[13] Anderl, R. \& Trippner, D. (2000). STEP STandard for the Exchange of Product Model Data: Eine Einführung in die Entwicklung, Implementierung und industrielle Nutzung der Normenreihe ISO 10303 (STEP). Stuttgart: Teubner. https://doi.org/10.1007/978-3-322-89096-2

[14] Pratt, M. J. (2001). Introduction to ISO 10303 - the STEP standard for product data exchange. In Journal of Computing and Information Science in Engineering, 1(1), 102-103. https://doi.org/10.1115/1.1354995
[15] SCRA. (2000). STEP Application Handbook. Retrieved from http://alvarestech.com/temp/capp/estudo_dirigido/cap p/www.ukceb.org/step/pages/step_application_handbook.p df

[16] ISO 10303-203:2011: Industrial automation systems and integration - Product data representation and exchange -Part 203: Application protocol: Configuration controlled $3 D$ design of mechanical parts and assemblies. Geneve: ISO

[17] ISO 10303-214:2010: Industrial automation systems and integration - Product data representation and exchange -Part 214: Application protocol: Core data for automotive mechanical design processes. Geneve: ISO

[18] ISO 10303-242:2014: Industrial automation systems and integration - Product data representation and exchange -Part 242: Application protocol: Managed model-based $3 D$ engineering. Geneve: ISO

[19] STEP AP242 Project. (2017, March 6). Retrieved from http://www.ap242.org/

[20] ProstepivipVerein. (2017, March 6). Retrieved from https://www.prostep.org/

[21] CAx Implementer Forum.(2017, March 6). Retrieved from https://www.cax-if.org/

[22] Kleinschrodt, C., Hackenschmidt, R., \& Rieg, F. (2017) Strategies for improving the quality of transferred 3D models in the context of the tool industry. In Croatian Association for PLM (Ed.), Management of Technology - Step to Sustainable Production. Dubrovnik.

\section{Contact information:}

Claudia KLEINSCHRODT, Dipl.-Ing.

University of Bayreuth

Lehrstuhl für Konstruktionslehre und CAD

Universitätsstraße 30, 95447 Bayreuth, Germany

claudia.kleinschrodt@uni-bayreuth.de

Frank RIEG, Prof. Dr.-Ing

University of Bayreuth

Lehrstuhl für Konstruktionslehre und CAD

Universitätsstraße 30, 95447 Bayreuth, Germany

konstruktionslehre@uni-bayreuth.de 